TRANSACTIONS OF THE

AMERICAN MATHEMATICAL SOCIETY

Volume 348, Number 1, January 1996

\title{
INVERTIBILITY PRESERVING LINEAR MAPS ON $\mathcal{L}(X)$
}

\author{
A. R. SOUROUR
}

\begin{abstract}
For Banach spaces $X$ and $Y$, we show that every unital bijective invertibility preserving linear map between $\mathcal{L}(X)$ and $\mathcal{L}(Y)$ is a Jordan isomorphism. The same conclusion holds for maps between $\mathbb{C} I+\mathcal{K}(X)$ and $\mathbb{C} I+\mathcal{K}(Y)$.
\end{abstract}

\section{INTRODUCTION AND STATEMENT OF MAIN RESULTS}

In this article, all vector spaces are over the complex field and all algebras are assumed to have an identity 1. The spectrum of an element $a$ is denoted by $\sigma(a)$. A linear map $\phi$ from an algebra $A$ to an algebra $B$ is called unital if $\phi(1)=1$ and is called invertibility preserving if $\phi(a)$ is invertible in $B$ for every invertible element $a \in A$. It is called an anti-homomorphism if $\phi(a b)=\phi(b) \phi(a)$ for every $a$ and $b \in A$, and a Jordan homomorphism if $\phi(a b+b a)=\phi(a) \phi(b)+\phi(b) \phi(a)$ for all $a$ and $b \in A$, or equivalently $\phi\left(a^{2}\right)=(\phi(a))^{2}$ for every $a \in A$. As usual, a bijective antihomomorphism (respectively Jordan homomorphism) is called an anti-isomorphism (respectively a Jordan isomorphism).

The algebra of all continuous linear operators on a Banach space or, more generally, a locally convex space $X$ is denoted by $\mathcal{L}(X)$, and the (nonunital) algebra of compact operators is denoted by $\mathcal{K}(X)$. The dual space of $X$ is denoted by $X^{\prime}$ and the adjoint of an operator $T$ is denoted by $T^{*}$. For $x \in X$ and $f \in E^{\prime}$, we denote by $x \otimes f$ the rank one operator on $X$ given by $u \rightarrow f(u) x$.

We now state our main result, characterizing bijective invertibility preserving linear maps between the algebras $\mathcal{L}(X)$ and $\mathcal{L}(Y)$ or the algebras $\mathbb{C} I+\mathcal{K}(X)$ and $\mathbb{C} I+\mathcal{K}(Y)$.

Theorem 1.1 (Main theorem). Let $X$ and $Y$ be Banach spaces over the complex field and let $\phi$ be a unital bijective linear map from $\mathcal{L}(X)$ onto $\mathcal{L}(Y)$ or from $\mathbb{C} I+$ $\mathcal{K}(X)$ onto $\mathbb{C} I+\mathcal{K}(Y)$. The following conditions are equivalent.

(a) $\phi$ preserves invertibility.

(b) $\phi$ is a Jordan isomorphism.

(c) $\phi$ is either an isomorphism or an anti-isomorphism.

(d) Either

(i) $Y$ is isomorphic to $X$ and $\phi(T)=A^{-1} T A$ for every $T \in \mathcal{L}(X)$, where $A$ is an isomorphism from $Y$ to $X$; or

Received by the editors October 26, 1993.

1991 Mathematics Subject Classification. Primary 47B48, 47B49; Secondary 47A10.

Key words and phrases. Invertibility preserving maps, Jordan homomorphism.

Supported in part by grants from the Natural Sciences and Engineering Research Council (Canada), and from the University of Victoria.

(C)1996 American Mathematical Society 
(ii) $Y$ is isomorphic to $X^{\prime}$ and $\phi(T)=B^{-1} T^{*} B$ for every $T \in \mathcal{L}(X)$, where $B$ is an isomorphism from $Y$ to $X^{\prime}$.

Remarks. 1. The map $\phi$ is not assumed to be continuous in any topology. The conclusion implies that if it preserves invertibility, then it must be continuous when $\mathcal{L}(X)$ and $\mathcal{L}(Y)$ are equipped with any of the standard operator topologies.

2. The equivalence of (b) and (c) is true for any additive map from a ring onto a prime ring [9, pp. 47-51]. We do not use this result here.

3. The equivalence of (c) and (d) includes the well-known result of Eidelheit [7] characterizing isomorphisms between $\mathcal{L}(X)$ and $\mathcal{L}(Y)$, and it also includes a characterization of anti-isomorphisms.

4. If $\mathcal{L}(X)$ is anti-isomorphic to $\mathcal{L}(Y)$, then both $X$ and $Y$ must be reflexive. Indeed, the form in (d)(ii) implies that every bounded operator on $X^{\prime}$ is the adjoint of an operator on $X$. Considering rank one operators $f \otimes G$ with $f \in X^{\prime}$ and $G \in X^{\prime \prime}$, we conclude that $X$ must be reflexive. Thus $X^{\prime}$, and hence also $Y$, are reflexive.

5. The case of a nonunital map $\phi$ can be reduced to the unital case by considering the map $\psi$ defined by $\psi(x)=\phi(1)^{-1} \phi(x)$. We state the conclusion formally.

Corollary 1.2. A bijective linear map $\phi: \mathcal{L}(X) \rightarrow \mathcal{L}(Y)$ preserves invertibility if and only if either $\phi(T)=A T B$ for every $T \in \mathcal{L}(X)$ or $\phi(T)=C T^{*} D$ for every $T \in \mathcal{L}(X)$, where $A: X \rightarrow Y, B: Y \rightarrow X, C: X^{\prime} \rightarrow Y$ and $D: Y \rightarrow X^{\prime}$ are bounded invertible operators.

The same conclusion holds for maps $\phi: \mathbb{C} I+\mathcal{K}(X) \rightarrow \mathbb{C} I+\mathcal{K}(Y)$.

We now indicate the place these results occupy among kindred results in the literature. The earliest result along these lines is that of Dieudonné [5] in which the finite dimensional case of the preceding corollary is obtained implicitly. (The article [5] deals with bijective "semilinear" maps on the algebra of square matrices over an arbitrary field that preserve noninvertibility.) A related result is obtained by Marcus and Purves [15] who again obtained the finite dimensional case of Corollary 1.2 for not necessarily bijective maps.

In the presence of commutativity, results of Gleason [8] and Kahane-Zelazko [11], refined by Zelazko [23], show that every unital invertibility preserving linear map from a Banach algebra $A$ to a semisimple commutative Banach algebra $B$ is multiplicative. (See also [16].)

Motivated by the results above, Kaplansky [12] asked when must additive invertibility preserving maps on rings be Jordan homomorphisms. Of course this is not always the case for arbitrary rings (see [12]). It is easy to construct a counterexample for linear unital maps on Banach algebras. Indeed if $A$ is obtained from a radical algebra by adjoining an identity, then any unital linear map on $A$ preserves invertibility, but is rarely multiplicative. We give a concrete example illustrating this phenomenon. Another example is given by Aupetit [1, p. 28]. More examples are given in $\S 3$ below.

Example 1. Let $A$ be the algebra of upper triangular $n \times n$ matrices with $n \geq 3$, and let $\phi\left(a_{i j}\right)=\left(b_{i j}\right)$ where $b_{12}=a_{13}, b_{13}=a_{12}$, and $b_{i j}=a_{i j}$ otherwise. It is immediate that $\phi$ is unital and preserves invertibility. However, if $c$ is the matrix given by $c_{13}=c_{23}=1$, and $c_{i j}=0$ otherwise, then $\phi\left(c^{2}\right)=0$ but $(\phi(c))^{2} \neq 0$, and so $\phi$ is not a Jordan homomorphism. 
Additional related results are in [1], [4], [10] and [18]. In [1, p. 30], Aupetit obtained a result which contains the Dieudonné-Marcus-Purves result and the Gleason-Kahane-Zelazko result. Articles [4] and [18] contain results on invertibility preserving positive linear maps on $C^{*}$-algebras and von Neumann algebras respectively. In [10], Jafarian and the author characterized spectrum preserving linear maps between $\mathcal{L}(X)$ and $\mathcal{L}(Y)$.

The foregoing results suggest the following question. It is tempting to conjecture a positive answer.

Question. Let $A$ and $B$ be semisimple Banach algebras and let $\phi$ be a unital bijective linear map from $A$ to $B$. If $\phi$ preserves invertibility, must it be a Jordan isomorphism?

The converse, however, is true under very general conditions as shown in the following proposition. As usual by a Jordan homomorphism between rings we mean an additive map $\phi$ satisfying $\phi(x \circ y)=\phi(x) \circ \phi(y)$, where $x \circ y=x y+y x$.

Proposition 1.3. Let $R$ and $R^{\prime}$ be two rings with identities 1 and $1^{\prime}$ respectively such that in $R^{\prime}$ the equation $2 x=0$ implies that $x=0$. If $\phi: R \rightarrow R^{\prime}$ is a Jordan homomorphism whose range contains $1^{\prime}$, then $\phi$ preserves invertibility.

Proof. First, we show that $\phi(1)=1^{\prime}$. By assumption, $1^{\prime}=\phi(u)$ for some $u \in R$, and so

$$
2 \cdot 1^{\prime}=\phi(u+u)=\phi(1 \circ u)=\phi(1) \circ \phi(u)=\phi(1) \circ 1^{\prime}=2 \phi(1) .
$$

Thus $\phi(1)=1^{\prime}$. Next, we note that

$$
4 x y x=2(y \circ x) \circ x-y \circ(x \circ x),
$$

and so

$$
\phi(x y x)=\phi(x) \phi(y) \phi(x),
$$

for all $x, y \in R$. In particular, if $a$ is an invertible element in $R$, then

$$
\phi(a)=\phi\left(a a^{-1} a\right)=\phi(a) \phi\left(a^{-1}\right) \phi(a) .
$$

Let $p_{1}=\phi(a) \phi\left(a^{-1}\right)$ and $p_{2}=\phi\left(a^{-1}\right) \phi(a)$. From equation (1), we have $p_{1}^{2}=p_{1}$ and $p_{2}^{2}=p_{2}$. On the other hand

$$
p_{1}+p_{2}=\phi(a) \circ \phi\left(a^{-1}\right)=\phi\left(a \circ a^{-1}\right)=2 \cdot \phi(1)=2 \cdot 1^{\prime} .
$$

Therefore $\left(2 \cdot 1^{\prime}-p_{1}\right)^{2}=2 \cdot 1^{\prime}-p_{1}$, which leads to $2\left(p_{1}-1^{\prime}\right)=0$ and so $p_{1}=p_{2}=1^{\prime}$. Thus $\phi(a)$ is invertible and $\phi\left(a^{-1}\right)=\phi(a)^{-1}$.

\section{Spectral characterization of Rank one operators}

In this section, we prove a characterization of rank one operators in terms of the spectrum, a result which may be of independent interest. The equivalence of conditions (i) and (ii) in the following theorem is given in [10] and is used there to characterize spectrum preserving maps. The stronger equivalence of (i) and (iii) is what is needed to prove our main results.

Theorem 2.1. For an operator $R \in \mathcal{L}(X)$, the following conditions are equivalent:

(i) $\operatorname{rank} R \leq 1$.

(ii) For every $T \in \mathcal{L}(X)$ and all distinct scalars $\alpha$ and $\beta$,

$$
\sigma(T+\alpha R) \cap \sigma(T+\beta R) \subseteq \sigma(T) .
$$


(ii) $^{\prime}$ Condition (*) is satisfied for every $T$ of rank at most 2.

(iii) For every $T \in \mathcal{L}(X)$, there exists a compact subset $K_{T}$ of the complex plane, such that

$$
\sigma(T+\alpha R) \cap \sigma(T+\beta R) \subseteq K_{T},
$$

for all scalars $\alpha \neq \beta$.

(iii)' Condition (**) is satisfied for every $T$ of rank at most 2.

We shall state and prove the equivalence of (i), (ii)', and (iii) ${ }^{\prime}$ for locally convex spaces. In particular, the main results hold when $X$ is a dual Banach space in its weak* topology and so $\mathcal{L}(X)$ consists of the weak ${ }^{*}$-continuous operators. We refer to [17] for basic results on locally convex spaces. The Hahn-Banach theorem [17, Theorem 3.6] for locally convex spaces will be implicitly used throughout.

Proposition 2.2. Let $E$ be a locally convex topological vector over the complex field and let $R \in \mathcal{L}(E)$. The following conditions are equivalent.

(i) $\operatorname{rank} R \leq 1$.

(ii)' $\sigma(T+\alpha R) \cap \sigma(T+\beta R) \subseteq \sigma(T)$ for every $T \in \mathcal{L}(E)$ of rank at most 2 and all scalars $\alpha$ and $\beta$ with $\alpha \neq \beta$.

(iii)' For every $T \in \mathcal{L}(E)$ of rank at most 2 , there exists a compact subset $K_{T}$ of the complex plane such that

$$
\sigma(T+\alpha R) \cap \sigma(T+\beta R) \subseteq K_{T},
$$

for all scalars $\alpha \neq \beta$.

Proof. We first show that (i) implies that (ii)' holds for arbitrary $T \in \mathcal{L}(E)$. Assume that $\operatorname{rank} R \leq 1$, that $T \in \mathcal{L}(E)$ and that $\alpha_{1}$ and $\alpha_{2} \in \mathbb{C}$ with $\alpha_{1} \neq \alpha_{2}$. Further, assume to the contrary that $\lambda \in \sigma\left(T+\alpha_{1} R\right) \cap \sigma\left(T+\alpha_{2} R\right)$ and $\lambda \notin \sigma(T)$. It follows that $\alpha_{1} \neq 0$ and $\alpha_{2} \neq 0$ and that $\lambda-T-\alpha_{j} R$ is not invertible for $j=1,2$. Therefore $I-\alpha_{j}(\lambda-T)^{-1} R$ is not invertible, and so $\frac{1}{\alpha_{1}}$ and $\frac{1}{\alpha_{2}}$ belong to the spectrum of the operator $(\lambda-T)^{-1} R$ which has rank at most 1 . This is impossible, and so the implication (i) $\Rightarrow$ (ii) is established.

The implication (ii) ${ }^{\prime} \Rightarrow\left(\right.$ (iii) $^{\prime}$ is obvious since the spectrum of a finite rank operator is a finite subset of the complex plane.

To prove that (iii) $\Rightarrow$ (i), assume that $R$ satisfies condition (iii)'. First, we take $T=0$, to conclude that $\sigma(R)$ contains at most one nonzero complex number. Indeed if $\sigma(R)$ contains two distinct nonzero complex numbers $\lambda$ and $\mu$, then every complex number $w$ belongs to $\sigma\left(\lambda^{-1} w R\right) \cap \sigma\left(\mu^{-1} w R\right)$, contradicting the assumption. Therefore there is a nonzero complex number $c$ such that $\sigma(T) \subseteq\{0, c\}$.

Next, we apply condition (iii)', with $\operatorname{rank} T=1$. Let $x \in E$ and $f \in E^{\prime}$ be two nonzero vectors and let $G(z):=f\left((1-z R)^{-1} x\right)$, for $z \in \mathbb{C} \backslash\left\{\frac{1}{c}\right\}$. We aim to apply condition (iii)' with $T=x \otimes f$ to conclude that the equation $G(z)=w$ has at most one solution for every $w$ with $|w|$ large enough. We start by showing that if $G(z)=w$, then $w \in \sigma(T+z w R)$, where $T=x \otimes f$. Indeed if $u=(1-z R)^{-1} x$, then $u \neq 0$, and

$$
(w I-T-z w R) u=w(1-z R) u-T u=w x-f(u) x=(w-G(z)) x=0,
$$

establishing that $w$ is an eigenvalue of $T+z w R$. If $w \notin K_{T}$ and if $G\left(z_{1}\right)=G\left(z_{2}\right)=$ $w$, then we have

$$
w \in \sigma\left(T+z_{1} w R\right) \cap \sigma\left(T+z_{2} w R\right)
$$


and so $z_{1}=z_{2}$. We have shown that, for $w$ large enough, the equation $G(z)=w$ has at most one solution.

Picard's "Big" Theorem [22, p. 283] asserts that, in a neighbourhood of an essential singularity, an analytic function assumes all values, with at most one possible exception, infinitely often. Consequently, the function $G$ has poles (or removable singularities) at each of $\frac{1}{c}$ and $\infty$. Thus $G$ must be a rational function. Indeed $G(z)=P(z) / Q(z)$ where $P$ and $Q$ are polynomials, $Q(z)=(c z-1)^{n}$ for some nonnegative integer $n$ and $P\left(\frac{1}{c}\right) \neq 0$. We will show that $\operatorname{deg} P \leq 1$ and $\operatorname{deg} Q \leq 1$. For all $w$ large enough, say $|w|>r$, we know that the equation

$$
P(z)-w Q(z)=0
$$

has at most one solution. If $\operatorname{deg}(P-w Q)$ is larger than 1 , then any solution of (1) must also satisfy the equation

$$
P^{\prime}(z)-w Q^{\prime}(z)=0
$$

It follows that, for any such $w$, the corresponding solution $z$ satisfies the polynomial equation

$$
Q^{\prime}(z) P(z)-Q(z) P^{\prime}(z)=0 .
$$

If the polynomials $Q^{\prime} P-P Q^{\prime}$ is identically zero, then $G$ is constant and we are done. If not, then equation (3) has only a finite number of solutions and so the polynomial $P-w Q$ has degree larger than 1 for only a finite number of values of $w$ among $\{w:|w|>r\}$, and so $P-w Q$ has degree at most 1 for all $w$ large enough. This implies that $\operatorname{deg} P \leq 1$ and $\operatorname{deg} Q \leq 1$. Hence

$$
G(z)=a z+b \quad \text { or } \quad \frac{a z+b}{c z-1}
$$

for some $a, b \in \mathbf{C}$.

Our next step is to use this form of $G$ to conclude that the operator $R$ satisfies a quadratic equation; indeed we show that $R^{2}=0$ or $R^{2}=c R$. The form of the function $G$ above implies that $G$ satisfies one of the following differential equations:

$$
G^{\prime \prime}(z)=0 \quad \text { or } \quad(c z-1) G^{\prime \prime}(z)+2 c G^{\prime}(z)=0 .
$$

In particular $G^{\prime \prime}(0)=0$ or $G^{\prime \prime}(0)=2 c G^{\prime}(0)$. Direct computation yields that $G^{\prime}(0)=f(R x)$ and $G^{\prime \prime}(0)=2 f\left(R^{2} x\right)$. We conclude that, for every $x \in E$ and every $f \in E^{\prime}$, either $f\left(R^{2} x\right)=0$ or $f\left(\left(R^{2}-c R\right) x\right)=0$. We show that this implies that $R^{2}=0$ or $R^{2}-c R=0$. First, for a fixed $x \in E$, we have $E^{\prime}$ equals the union of the two subspaces $\left\{f \in E^{\prime}: f\left(R^{2} x\right)=0\right\}$ and $\left\{f \in E^{\prime}: f\left(\left(R^{2}-R\right) x\right)=0\right\}$ and so one of the subspaces must be all of $E^{\prime}$, implying that $R^{2} x=0$ or $\left(R^{2}-c R\right) x=0$. A similar argument shows that one of the latter equations is satisfied for every $x \in E$ and hence $R^{2}=0$ or $R^{2}=c R$.

We are now in a position to prove that $R$ has rank one. We consider the two cases $R^{2}=c R$ and $R^{2}=0$ separately. If $R^{2}=c R$ and if $\operatorname{rank} R>1$, let $u$ and $v$ be two linearly independent vectors in the range of $R$. Thus $R u=c u$ and $R v=c v$. Let $f \in E^{\prime}$ be such that $f(u)=0$ and $f(v)=1$ and let $T=v \otimes f$. If $\lambda$ is any complex number, then

$$
\lambda \in \sigma\left(T+\frac{\lambda}{c} R\right) \cap \sigma\left(T+\frac{(\lambda-1)}{c} R\right)
$$


since $\left(T+\frac{\lambda}{c} R\right) u=\lambda u$ and $\left(T+\frac{(\lambda+1)}{c} R\right) v=\lambda v$. This contradicts condition (iii)'. Therefore $\operatorname{rank} R \leq 1$.

Next, we consider the case $R^{2}=0$. If $\operatorname{rank} R \geq 2$, let $u_{2}$ and $u_{4}$ be linearly independent vectors in range $R$ and let $u_{1}$ and $u_{3} \in X$ be such that $R u_{1}=u_{2}$ and $R u_{3}=$ $u_{4}$. Thus $u_{1}, u_{2}, u_{3}, u_{4}$ are linearly independent and $W:=\operatorname{span}\left\{u_{1}, u_{2}, u_{3}, u_{4}\right\}$ is a subspace invariant under $R$ with $R \mid W$ having matrix representation

$$
\left(\begin{array}{ll}
0 & 0 \\
1 & 0
\end{array}\right) \oplus\left(\begin{array}{ll}
0 & 0 \\
1 & 0
\end{array}\right)
$$

relative to the basis $\left\{u_{1}, u_{2}, u_{3}, u_{4}\right\}$. Let $f_{2}$ and $f_{4} \in E^{\prime}$ be such that $f_{i}\left(u_{j}\right)=1$ if $i=j$ and 0 if $i \neq j$ and define a rank two operator $T$ by $T=u_{1} \otimes f_{2}+4 u_{3} \otimes f_{4}$. Thus $T$ leaves $W$ invariant and $T \mid W$ has matrix representation

$$
\left(\begin{array}{ll}
0 & 1 \\
0 & 0
\end{array}\right) \otimes\left(\begin{array}{ll}
0 & 4 \\
0 & 0
\end{array}\right)
$$

Every complex number $\lambda$ belongs to $\sigma\left(T+\lambda^{2} R\right) \cap \sigma\left(T+\frac{\lambda^{2}}{4} R\right)$. The contradiction establishes the desired conclusion, i.e., $\operatorname{rank} R \leq 1$.

Proof of Theorem 2.1. The equivalence of (i), (ii) ${ }^{\prime}$ and (iii) ${ }^{\prime}$ is a special case of Proposition 2.2. The implication (i) $\Rightarrow$ (ii) is proved above. The implication (ii) $\Rightarrow$ (iii) follows from the fact that the spectrum of every bounded operator is a compact subset of the complex plane, and the implication (iii) $\Rightarrow$ (iii) ${ }^{\prime}$ is trivial.

Remark. From the above proof, it follows that if $R^{2} \neq 0$, and if condition $(*)$ or (**) of Theorem 2.1 is satisfied for operators $T$ of $\operatorname{rank} \leq 1$, then $\operatorname{rank} R \leq 1$. The finite dimensional case of this observation is in [5].

We state the following variants of the above results.

Proposition 2.3. The conclusions of Theorem 2.1 and Proposition 2.2 remain true if the spectrum is replaced by the point spectrum (i.e., the set of eigenvalues).

Proof. Omitted.

Proposition 2.4. With the same notation as in Theorem 2.1 and Proposition 2.2, the following are equivalent.

(i) $R$ is a scalar or $\operatorname{rank} R \leq 1$.

(ii) Condition $(*)$ is satisfied for every nilpotent operator $T$ with $T^{2}=0$.

(iii) Condition $(* *)$ is satisfied for every nilpotent operator $T$ with $T^{2}=0$.

Proof. We only indicate the minor changes required in the proof of Proposition 2.2. The rank one operator $T=x \otimes f$ used in that proof will be assumed to satisfy $T^{2}=0$, i.e. $f(x)=0$. We conclude that if $f(x)=0$, then either $f\left(R^{2} x\right)=0$ or $f\left(\left(R^{2}-c R\right) x\right)=0$. It follows that either $R^{2} x=\lambda_{x} x$ or $\left(R^{2}-c R\right) x=\mu_{x} x$ for scalars $\lambda_{x}$ and $\mu_{x}$; consequently either $R^{2}$ is a scalar or $R^{2}-c R$ is a scalar. Since $\sigma(R) \subseteq\{0, c\}$, we have $R$ satisfying one of the equations $R^{2}=0, R^{2}=c^{2} I$ or $R^{2}-c R=0$. The first case is dealt with in Proposition 2.2. The second case, $R^{2}=c^{2} I$, implies that $R=c I$ since $-c \notin \sigma(R)$. Finally if $R^{2}=c R$ and if $R$ is not a scalar or of rank one, then

$$
R=\left[\begin{array}{lll}
0 & 0 & 0 \\
0 & c & 0 \\
0 & 0 & c
\end{array}\right] \oplus R_{1}
$$


for some operator $R_{1}$. If

$$
T=\left[\begin{array}{ccc}
1 & -1 & 0 \\
1 & -1 & 0 \\
0 & 0 & 0
\end{array}\right] \oplus 0,
$$

then $T^{2}=0$, and every complex number $\lambda$ belongs to

$$
\sigma\left(T+\frac{\lambda}{c} R\right) \cap \sigma\left(T+\frac{\lambda^{2}}{c(\lambda-1)} R\right) .
$$

\section{PROOF OF THE MAIN THEOREM}

In this section, we shall prove the main theorem. We first show that invertibility preserving maps are rank-reducing.

Lemma 3.1. Let $A, B$ be two unital algebras and $\phi: A \rightarrow B$ a unital linear map. If $\phi$ preserves invertibility, then $\sigma(\phi(a)) \subseteq \sigma(a)$ for every $a \in A$.

Proof. Obvious.

Lemma 3.2. Let $X$ be a Banach space, $Y$ a locally convex space and let $\mathcal{S}$ be a subspace of $\mathcal{L}(X)$ containing all finite rank operators and containing the identity. If $\phi: \mathcal{S} \rightarrow \mathcal{L}(Y)$ is a linear map which preserves invertibility and if range $\phi$ contains all operators on $Y$ of finite rank, then $\operatorname{rank} \phi(T) \leq \operatorname{rank} T$ for every $T \in \mathcal{S}$.

Proof. We may assume without loss of generality that $\phi(I)=I$, since we may replace $\phi$ by the map $\psi$ given by $\psi(T)=\phi(I)^{-1} \phi(T)$. If $R \in \mathcal{L}(X)$ and $\operatorname{rank} R=1$, then condition $(*)$ of Theorem 2.1 is satisfied. This, together with the spectral inclusion of Lemma 3.1, implies that

$$
\sigma(\phi(T)+\alpha \phi(R)) \cap \sigma(\phi(T)+\beta \phi(R)) \subseteq \sigma(T)
$$

for $\alpha \neq \beta$. Since $\sigma(T)$ is a compact subset of the plane, and since the range of $\phi$ contains every operator of rank at most 2 , we may use Proposition 2.2 to conclude that $\operatorname{rank} \phi(R) \leq 1$. If $T \in \mathcal{L}(X)$ with $\operatorname{rank} T=n<\infty$, then $T$ can be written as a sum of $n$ operators of rank one. Thus $\phi(T)$ is a sum of $n$ operators, each having rank at most 1 , and so $\operatorname{rank} \phi(T) \leq n$. If $\operatorname{rank} T=\infty$, there is nothing to prove.

To prove Theorem 1.1, it suffices to prove that (a) implies (d) since the implications $(\mathrm{d}) \Rightarrow(\mathrm{c}) \Rightarrow(\mathrm{b})$ are trivial and $(\mathrm{b}) \Rightarrow$ (a) follows from Proposition 1.3. The implication (a) $\Rightarrow$ (d) is contained in Theorem 3.4 below. First, we need a lemma.

Lemma 3.3. Let $E$ be a vector space, $x \in E, f$ a linear functional on $E$ and $T$ an invertible linear transformation from $E$ to $E$. The transformation $T-x \otimes f$ is invertible if and only if $f\left(T^{-1} x\right) \neq 1$. If $f\left(T^{-1} x\right) \neq 1$, then $T-x \otimes f$ is invertible in every unital subalgebra of $\mathcal{L}(E)$ that contains $T, T^{-1}$ and $T-x \otimes f$.

Proof. Since $T-x \otimes f$ is invertible if and only if $T^{-1}(T-x \otimes f)$ is, it suffices to show that $I-u \otimes f$ is invertible if and only if $f(u) \neq 1$. If $f(u)=1$, then $u \neq 0$ and $(I-u \otimes f) u=0$, and so $I-u \otimes f$ is not invertible. Conversely if $f(u) \neq 1$, we get, by direct computation, that

$$
(I-u \otimes f)^{-1}=\left(I+(1-f(u))^{-1} u \otimes f\right),
$$

which gives an inverse of $I-u \otimes f$ in every algebra containing $I$ and $I-$ $u \otimes f$. 
In the proof of the next result, we shall employ the notation $\langle\cdot, \cdot\rangle$ to denote the duality between a topological vector space and its dual. We consider maps from a subalgebra $\mathcal{A}$ of $\mathcal{L}(X)$ into $\mathcal{L}(Y)$. When we say that such a map $\phi$ preserves invertibility, we mean that if $T$ is invertible in $\mathcal{A}$, then $\phi(T)$ is invertible in $\mathcal{L}(Y)$.

Theorem 3.4. Let $X$ be a Banach space, $Y$ a locally convex space, $\mathcal{A}$ a unital subalgebra of $\mathcal{L}(X)$ containing all operators of finite rank, and $\phi: \mathcal{A} \rightarrow \mathcal{L}(Y)$ a linear map preserving invertibility whose range contains every finite rank operator on $Y$. It follows that either $\phi(F)=0$ for every finite rank operator $F \in \mathcal{L}(X)$, or $\phi$ is injective. In the latter case, either:

(i) there exist bijective linear transformations $A: Y \rightarrow X$ and $B: X \rightarrow Y$ with A weak-weak continuous such that

$$
\phi(T)=B T A
$$

for every $T \in \mathcal{A}$; or

(ii) there exist bijective linear transformations $A: Y \rightarrow X^{\prime}$ and $B: X^{\prime} \rightarrow Y$ with A weak-weak* continuous such that

$$
\phi(T)=B T^{*} A
$$

for every $T \in \mathcal{A}$.

Remarks. 1. Of course the conclusion of Theorem 3.4 would also follow from the stronger hypothesis that $\phi(T)$ is invertible for every $T \in \mathcal{A}$ which has an inverse in $\mathcal{L}(X)$.

2. If $\phi(I)=I$, then $B=A^{-1}$.

3. If $Y$ is a Banach space, then the weak continuity of the operator $A$ and the closed graph theorem imply that $A$ is bounded. The open mapping theorem implies that $A^{-1}$ is bounded and hence $B$ is also bounded since $B=\phi(I) A^{-1}$.

4. We do not know of any example where $\phi$ annihilates finite rank operators. It is conceivable that the map $\phi$ in Theorem 3.4 is always injective. Indeed this is the case when $X$ and $Y$ are Hilbert spaces as shown in $\S 6$ below.

Proof. Let $x \in X$ and $f \in X^{\prime}$. By Lemma 3.2, $\operatorname{rank} \phi(x \otimes f) \leq 1$, and so $\phi(x \otimes f)=$ $y \otimes g$ for some $y \in Y$ and $g \in Y^{\prime}$. Unless $y \otimes g=0$, the vectors $y$ and $g$ are determined by $x$ and $f$ up to a multiplicative constant. By a standard argument (see [7, p. 259]), the multiplicative constant can be chosen in such a way that $y$ depends only on one of the variables $x$ or $f$ and that $g$ depends only on the other variable; i.e., either

$$
\phi(x \otimes f)=B x \otimes C f
$$

where $B: X \rightarrow Y$ and $C: X^{\prime} \rightarrow Y^{\prime}$ are linear transformations, or

$$
\phi(x \otimes f)=R f \otimes S x
$$

where $R: X^{\prime} \rightarrow Y$ and $S: X \rightarrow Y^{\prime}$ are linear transformations.

We start by assuming that equation (1) is satisfied. We may also assume, with no loss of generality, that $\phi(I)=I$. For an arbitrary operator $T \in \mathcal{A}$, we have

$$
\phi(I-z T-x \otimes f)=I-z \phi(T)-B x \otimes C f .
$$

If $|z|<r:=\|T\|^{-1}$, then $I-z T$ is invertible in $\mathcal{A}$, and hence $I-z \phi(T)$ is invertible in $\mathcal{L}(Y)$. Let

$$
F_{x, f}(z):=\left\langle(I-z T)^{-1} x, f\right\rangle,
$$


and

$$
G_{x, f}(z):=\left\langle(I-z \phi(T))^{-1} B x, C f\right\rangle .
$$

If $F_{x, f}(z) \neq 1$, then by Lemma 3.3 the operator $I-z T-x \otimes f$ is invertible in $\mathcal{A}$, and so $I-z \phi(T)-B x \otimes C f$ is invertible in $\mathcal{L}(Y)$, implying that $G_{x, f}(z) \neq 1$. Equivalently, the equation $G_{x, f}(z)=1$ implies that $F_{x, f}(z)=1$. But the function $F_{x, f}$ and $G_{x, f}$ are linear in the parameters $x$ and $f$ and so if $G_{x, f}(x)=w \neq 0$, then $F_{x, f}(z)=w$. By analyticity of the two functions, we see that $F_{x, f} \equiv G_{x, f}$ unless $G_{x, f} \equiv 0$. We will show that unless $G_{u, g} \equiv 0$ for every $u \in X$ and every $g \in X^{\prime}$, we have $F_{x, f} \equiv G_{x, f}$ for every $x \in X$ and $f \in X^{\prime}$. Indeed if $G_{x_{1}, f_{1}} \not \equiv 0$, then by the foregoing argument

$$
X^{\prime}=\left\{f: G_{x_{1}, f} \equiv 0\right\} \cup\left\{f: F_{x_{1}, f} \equiv G_{x_{1}, f}\right\},
$$

a union of two subspaces. Thus one of the two subspaces is all of $X^{\prime}$. Since $G_{x_{1}, f_{1}} \not \equiv 0$, then

$$
\left\{f: F_{x_{1}, f} \equiv G_{x_{1}, f}\right\}=X^{\prime}
$$

i.e.

$$
F_{x_{1}, f} \equiv G_{x_{1}, f} \quad \text { for all } f \in X^{\prime}
$$

Similarly

$$
F_{x, f_{1}} \equiv G_{x, f_{1}} \quad \text { for all } x \in X .
$$

Finally, considering $F_{x+x_{1}, f+f_{1}}$ and linearity, we get that $F_{x, f} \equiv G_{x, f}$ for all $x \in X$ and $f \in X^{\prime}$. Thus we have shown that, for every $T \in \mathcal{A}$, either

$$
\left\langle(I-z T)^{-1} x, f\right\rangle=\left\langle(I-z \phi(T))^{-1} B x, C f\right\rangle
$$

for all $x \in X, f \in X^{\prime}$ and $z \in\{\zeta \in \mathbb{C}:|\zeta|<r\}$, or

$$
\left\langle(I-z \phi(T))^{-1} B x, C f\right\rangle=0
$$

for all $x \in X, f \in X^{\prime}$ and $z \in\{\zeta \in \mathbb{C}:|\zeta|<r\}$.

Taking derivatives at 0 , we obtain that, for every $T \in \mathcal{A}$, either

$$
\langle T x, f\rangle=\langle\phi(T) B x, C f\rangle
$$

for all $x \in X$ and $f \in X^{\prime}$, or

$$
\langle\phi(T) B x, C f\rangle=0
$$

for every $x \in X$ and $f \in X^{\prime}$. Thus $\mathcal{A}$ is a union of two subspaces corresponding to the two alternatives above, and so one of the alternatives (3) or (4) is satisfied for all $T \in \mathcal{A}$. If equation (4) is satisfied, then by taking $\phi(T)=y \otimes g$ for arbitrary $y \in Y$ and $g \in Y^{\prime}$ we have $g(B x) \cdot\langle y, C f\rangle=0$ for every $x \in X, y \in Y, f \in X^{\prime}$ and $g \in Y^{\prime}$. This easily implies that $B=0$ or $C=0$ and so $\phi(x \otimes f)=B x \otimes C f=0$. Therefore $\phi(F)=0$ for every finite rank operator $F \in \mathcal{L}(X)$.

If equation (3) is satisfied, then for every $y \in Y$, there exist $T_{y} \in \mathcal{L}(X)$ and $x_{y} \in X$ such that $\phi\left(T_{y}\right) B x_{y}=y$ and hence $\langle y, C f\rangle=\left\langle T_{y} x_{y}, f\right\rangle$. This shows that there exists a linear transformation $A: Y \rightarrow X$ such that

$$
\langle A y, f\rangle=\langle y, C f\rangle .
$$

In other words, $C=A^{*}$. Now equation (3) can be written in the form

$$
\langle T x, f\rangle=\langle A \phi(T) B x, f\rangle,
$$


for every $x \in X$ and $f \in X^{\prime}$. Thus

$$
T=A \phi(T) B
$$

for all $T \in \mathcal{A}$. It follows immediately that $\phi$ is injective.

We will show that $A$ and $B$ are bijective. The surjectivity of $A$ follows immediately from equation (6). To prove the injectivity of $A$, assume that $A y=0$ for some $y \in Y$. Let $T_{1} \in \mathcal{A}$ be such that $\phi\left(T_{1}\right)=y \otimes g$ with $g \in Y^{\prime}, g \neq 0$. Therefore $A \phi\left(T_{1}\right)=0$, and by equation (6), $T_{1}=0$. Hence $y \otimes g=\phi\left(T_{1}\right)=0$ and so $y=0$. This proves that $A$ is injective. Taking $T=I$ in equation (6) gives $A B=I$, and since $A$ is injective, this implies that $B A=I$, i.e., $B=A^{-1}$, and hence $\phi(T)=B T A$.

The weak-weak continuity of $A$ follows easily from equation (5).

We now consider the case where equation (2), rather than equation (1), is satisfied. By a similar argument, we have that either $\phi(F)=0$ for all finite rank operators $F$ or there exist linear transformations $R: X^{\prime} \rightarrow Y$ and $S: X \rightarrow Y^{\prime}$ such that

$$
\langle T x, f\rangle=\langle\phi(T) R f, S x\rangle
$$

for all $x \in X, f \in X^{\prime}$ and $T \in \mathcal{A}$. Let $y \in Y$. Since the range of $\phi$ contains every rank one operator, there exist $f_{y} \in X^{\prime}$ and $T_{y} \in \mathcal{L}(X)$ such that $\phi\left(T_{y}\right) R f_{y}=y$, and so

$$
\langle y, S x\rangle=\left\langle T_{y} x, f_{y}\right\rangle=\left\langle x, T_{y}^{*} f_{y}\right\rangle .
$$

Therefore there exists a linear transformation $U: Y \rightarrow X^{\prime}$ such that

$$
\langle y, S x\rangle=\langle x, U y\rangle .
$$

Equation $\left(5^{\prime}\right)$ implies that $U$ is weak-weak* continuous, and that equation $\left(3^{\prime}\right)$ can be written in the form

$$
\left\langle x, T^{*} f\right\rangle=\langle x, U \phi(T) R f\rangle,
$$

and so

$$
T^{*}=U \phi(T) R .
$$

Now the same argument as before establishes that $U$ and $R$ are bijective, that $R=U^{-1}$ and that

$$
\phi(T)=R T^{*} U .
$$

This ends the proof of Theorem 3.4.

Remark. In all of our results, the condition on the range of $\phi$ cannot be removed as indicated by Example 2 below. As indicated earlier, we do not know whether the injectivity assumption in Theorem 1.1 can be removed (see also $\S 6$ below).

Example 2. Let $H$ be a Hilbert space and let $f$ be a nonzero linear functional on $\mathcal{L}(H)$ such that $f(I)=0$. Define $\phi: \quad \mathcal{L}(H) \rightarrow \mathcal{L}(H \oplus H)$ by

$$
\phi(T)=\left[\begin{array}{cc}
T & f(T) I \\
0 & T
\end{array}\right] .
$$

It follows that $\phi(I)=I$, that $\phi$ preserves invertibility (indeed $\phi$ preserves the spectrum; equivalently $\phi$ preserves invertibility in both directions). But $\phi$ is not a Jordan homomorphism as can be easily seen. 
Remark. Example 2 above shows that invertibility preserving linear maps need not be continuous. Of course if such a map is also bijective, then it is continuous by Theorem 1.1. See also $\S 4$ below.

\section{Continuity}

It follows from Theorem 1.1 that a bijective invertibility preserving linear map from $\mathcal{L}(X)$ to $\mathcal{L}(Y)$ is norm-continuous. We give a direct proof of continuity without assuming injectivity of $\phi$. We shall however assume that $\phi$ is surjective. (The proof requires only that range $\phi$ contains all finite rank operators.) Example 2 above shows that such conditions cannot be removed.

Theorem 4.1. Let $X$ and $Y$ be Banach spaces. If $\phi: \mathcal{L}(X) \rightarrow \mathcal{L}(Y)$ is a surjective invertibility preserving linear map, then it is norm continuous.

Proof. By the closed graph theorem, it suffices to prove that if $T_{n} \in \mathcal{L}(X), T_{n} \rightarrow 0$ and $\phi\left(T_{n}\right) \rightarrow S$, then $S=0$. Let $S_{n}=\phi\left(T_{n}\right), \varepsilon_{n}=\left\|T_{n}\right\|$ and denote the spectral radius of an operator $A$ by $r(A)$. For every $T \in \mathcal{L}(X)$, and every scalar $\alpha$, we have

$$
r\left(T+\alpha T_{n}\right) \leq\left\|T+\alpha T_{n}\right\| \leq\|T\|+|\alpha| \varepsilon_{n} .
$$

We may assume without loss of generality that $\phi(I)=I$, and so $\phi$ satisfies the spectral inclusion of Lemma 3.1. Thus

$$
r\left(\phi(T)+\alpha S_{n}\right) \leq\|T\|+|\alpha| \varepsilon_{n} .
$$

Since $\phi$ is surjective, we conclude that, for every operator $R \in \mathcal{L}(Y)$, there exists a positive number $\rho=\rho_{R}$ such that

$$
r\left(R+\alpha S_{n}\right) \leq \rho+|\alpha| \varepsilon_{n} .
$$

We will apply equation (1) to operators $R$ of the form $y \otimes g$. Toward this end let $y \in Y$ and $g \in Y^{\prime}$ be nonzero vectors, let $R=y \otimes g$ and let

$$
G_{n}(z):=\left\langle\left(1-z S_{n}\right)^{-1} y, g\right\rangle
$$

for $z \in \Omega_{n}$, where

$$
\Omega_{n}:=\left\{z:|z|<\varepsilon_{n}^{-1}\right\}
$$

and

$$
G(z):=\left\langle(1-z S)^{-1} y, g\right\rangle
$$

for $z \in \Omega$, where

$$
\Omega:=\left\{z: \frac{1}{z} \notin \sigma(S)\right\} .
$$

First, we claim that $G_{n}\left(\frac{1}{2} \Omega_{n}\right) \subseteq\{\zeta:|\zeta| \leq 2 \rho\}$. To prove this, let $z \in \mathbb{C}$ with $|z|<\left(2 \varepsilon_{n}\right)^{-1}$ and let $w=G_{n}(z)$. It follows that

$$
\left(w-\left(R+z w S_{n}\right)\right)\left(1-z S_{n}\right)^{-1} y=0,
$$

and hence $w \in \sigma\left(R+z w S_{n}\right)$. By equation (1), we get that $|w| \leq \rho+|z||w| \varepsilon_{n} \leq$ $\rho+\frac{1}{2}|w|$, which implies that $|w| \leq 2 \rho$.

Next, we observe that for every $z \in \Omega, z$ belongs to $\Omega_{n}$ for $n$ large enough and that $G_{n}(z) \rightarrow G(z)$. Indeed it is not hard to prove that $G_{n} \rightarrow G$ uniformly on compact subsets of $\Omega$. 
Now if $k>0$ and if $B_{k}:=\{z:|z| \leq k\}$, then, for all $n$ large enough, we have $B_{k} \subseteq \frac{1}{2} \Omega_{n}$ and so $G_{n}\left(B_{k}\right) \subseteq B_{2 \rho}$. Upon taking limits, we get $G\left(B_{k} \cap \Omega\right) \subseteq B_{2 \rho}$. Since this is true for every $k>0$, we conclude that $G$ is bounded in $\Omega$; i.e.

$$
\left|\left\langle(1-z S)^{-1} y, g\right\rangle\right| \leq M_{y, g} \quad \text { for } z \in \Omega,
$$

where $M_{y, g}$ is a positive constant. By the uniform boundedness principle, there exists a positive constant $M$ such that

$$
\left\|(1-z S)^{-1}\right\| \leq M \quad \text { for } z \in \Omega .
$$

If $\zeta \in \partial \Omega$ and $z_{n} \in \Omega$ with $z_{n} \rightarrow \zeta$, then $\left\|\left(1-z_{n} S\right)^{-1}\right\|$ is bounded which is impossible [2, Theorem 14, p. 13]. Thus $\partial \Omega$ must be empty and so $\Omega=\mathbb{C}$. The function $(1-z S)^{-1}$ is a bounded entire function and hence, by Liouville's Theorem, $S=0$.

\section{Spectral Characterization of COMPaCtNess}

The following is a spectral characterization of compactness for operators in separable infinite dimensional Hilbert space. It will be used in $\S 6$ to establish an injectivity result.

Theorem 5.1. Let $H$ be a separable infinite dimensional Hilbert space and let $A \in$ $\mathcal{L}(H)$. The following conditions are equivalent:

(i) $A$ is compact.

(ii) $\sigma(T+A) \cap \sigma(T)$ is nonempty for every $T \in \mathcal{L}(H)$.

(iii) $\sigma(T+A) \cap \sigma(T)$ is nonempty for every nilpotent $T \in \mathcal{L}(H)$ satisfying $T^{3}=0$.

Proof. If $A$ is compact, then $\sigma(T+A) \cap \sigma(T)$ includes the essential spectrum of $T$ which is nonempty. It remains to show that if $A$ is noncompact, then there exists an operator $T$ such that $T^{3}=0$ and $T+A$ is invertible. We shall consider two cases according as $A$ is a scalar + compact or not.

First if $A$ is not a scalar + compact, then, by [3], $A$ is similar to an operator on $H \oplus H \oplus H$ having matrix representation

$$
\left[\begin{array}{lll}
0 & B & C \\
0 & D & E \\
I & F & G
\end{array}\right]
$$

We shall assume that $A$ equals such a matrix. Let

$$
T=\left[\begin{array}{ccc}
0 & I-B & -C \\
0 & 0 & I-E \\
0 & 0 & 0
\end{array}\right]
$$

It follows that $T^{3}=0$ and that

$$
(T+A)^{-1}=\left[\begin{array}{ccc}
G D-F & -G & I \\
I & 0 & 0 \\
-D & I & 0
\end{array}\right] .
$$

Next, we consider the case where $A$ is the sum of a nonzero scalar and a compact operator. By the Riesz decomposition of operators and the Jordan decomposition of nilpotent matrices, we may assume that

$$
A=N_{1} \oplus \cdots \oplus N_{m} \oplus(0 \oplus B)
$$


where each $N_{j}$ is a nonzero nilpotent Jordan cell, the operator 0 acts on a space of finite dimension $k$ and $B$ is invertible (the integers $m$ or $k$ may possibly be 0 ). Thus it suffices to prove the existence of the operator $T$ for the cases: $A=N_{j}$ and $A=0 \oplus B$.

When $A=N$, a nonzero nilpotent $n \times n$ Jordan cell, we take $T=\left(N^{t}\right)^{n-1}$. It follows easily that $T^{2}=0$ and that $T+A$ is invertible. If $A$ is invertible, we take $T=0$. If $A=\left[\begin{array}{ll}0 & 0 \\ 0 & B\end{array}\right]$ on $H_{1} \oplus H_{2}$ with $\operatorname{dim} H_{1}<\infty$ and $\operatorname{dim} H_{2}=\infty$, we take $T=\left[\begin{array}{cc}I & J \\ -L & -J L\end{array}\right]$, where $J: H_{1} \rightarrow H_{2}$ is an injective operator, and $L: H_{2} \rightarrow H_{1}$ is a left-inverse of $J$; i.e., $L J=I$ on $H_{1}$. By direct computation, we get that $T^{2}=0$ and

$$
(T+A)^{-1}=\left[\begin{array}{cc}
I-L B^{-1} J & L B^{-1} \\
-B^{-1} J & B^{-1}
\end{array}\right] .
$$

This ends the proof.

It is natural at this point to examine the implications of condition (ii) of the above theorem in the case of finite dimensional spaces. We show that it characterizes zero operators. We shall prove this for matrices over arbitrary fields.

Proposition 5.2. Let $A$ be an $n \times n$ matrix over an arbitrary field $F$. The following conditions are equivalent:

(i) $A=0$.

(ii) $\sigma(T+A) \cap \sigma(T)$ is nonempty for every $T \in M_{n}(F)$.

(iii) $0 \in \sigma(T+A)$ for every nilpotent $T \in M_{n}(F)$.

Proof. We need only show that if $A \neq 0$, then there exists a nilpotent matrix $N$ such that $A+N$ is invertible. We use induction on $n$. The ideas we use here are somewhat similar to the ideas in [21].

We start with $n=2$. If $A$ is a scalar, we may take $N=0$. If $A$ is nonscalar, then $A$ is similar to a matrix of the form $\left[\begin{array}{ll}0 & a \\ 1 & b\end{array}\right]$ and so we may assume that $A$ equals such a matrix. If we take $\left[\begin{array}{cc}0 & 1-a \\ 0 & 0\end{array}\right]$, then $N^{2}=0$ and $A+N$ is invertible.

Assume that $n \geq 3$ and that the result is true for all matrices of size less than $n$ and let $A$ be a nonzero $n \times n$ matrix. If $A$ is a scalar, then we may again take $N=0$. If $A$ is nonscalar, then we may, by using similarity, assume that

$$
A=\left[\begin{array}{cc}
1 & y^{t} \\
x & A_{0}
\end{array}\right]
$$

where $A_{0} \in M_{n-1}(F), x$ and $y \in F^{(n-1)}$ and where $x \neq 0$. We now show that, by taking another similarity if necessary, we may assume that $A_{0} \neq 0$. If $A_{0}=0$, let $z \in F^{(n-1)}$ be such that $z \neq 0$, but $z^{t} x=0$. Since

$$
\left[\begin{array}{cc}
1 & -z^{t} \\
0 & I
\end{array}\right]\left[\begin{array}{cc}
1 & y^{t} \\
x & 0
\end{array}\right]\left[\begin{array}{cc}
1 & z^{t} \\
0 & I
\end{array}\right]=\left[\begin{array}{cc}
1 & y^{t}+z^{t} \\
x & x z^{t}
\end{array}\right]
$$

and since $x z^{t} \neq 0$, we have shown that the matrix $A_{0}$ in equation (1) can be assumed to be nonzero. Now, by the induction hypothesis, there is an $(n-1) \times(n-1)$ nilpotent matrix $N_{0}$ such that $N_{0}+A_{0}$ is invertible. If we take

$$
N=\left[\begin{array}{cc}
0 & -y^{t} \\
0 & N_{0}
\end{array}\right]
$$

then $N$ is nilpotent and $N+A$ is invertible. 
Remark. In Proposition 5.2, unlike the situation in Theorem 5.1, we cannot always take the nilpotent matrix $N$ to have index of nilpotency at most 3 . Indeed if $A$ is an $n \times n$ matrix of rank one and if $N$ is nilpotent and $A+N$ is invertible, then $\operatorname{rank} N=n-1$, and so $N$ must be similar to a Jordan cell; hence the index of nilpotency of $N$ is $n$.

\section{INJECTIVITY}

If $X$ is a separable Hilbert space, we shall show that Theorem 1.1 remains true if we assume that $\phi$ is surjective but do not assume that it is injective. We prove that injectivity is part of the conclusion.

Theorem 6.1. Let $H$ be a separable Hilbert space and $Y$ a Banach space. If $\phi: \quad \mathcal{L}(H) \rightarrow \mathcal{L}(Y)$ is an invertibility preserving linear map whose range contains the finite rank operators on $Y$, then $\phi$ is one-to-one.

Proof. Again, we assume that $\phi(I)=I$ so that, by Lemma 3.1, $\phi$ satisfies the spectral inclusion

$$
\sigma(\phi(T)) \subseteq \sigma(T) .
$$

If $\phi$ is not one-to-one, then, by Theorem 3.4, $\phi(F)=0$ for every finite rank operator $F$ and, by continuity (Theorem 4.1 ),$\phi(K)=0$ for every compact operator $K \in$ $\mathcal{L}(H)$.

On the other hand, if $R_{1}$ is a rank one operator in $\mathcal{L}(Y)$, then there exists an $R \in \mathcal{L}(H)$ such that $R_{1}=\phi(R)$. For every $S \in \mathcal{L}(Y)$, we have

$$
\sigma(S+\phi(R)) \cap \sigma(S) \neq \varnothing,
$$

since the intersection includes the essential spectrum of $S$. If $T \in \mathcal{L}(H)$, then equations (1) and (2) imply that $\sigma(T+R) \cap \sigma(T)$ is nonempty. By Theorem 5.1, we conclude that $R$ is a compact operator, and hence $R_{1}=\phi(R)=0$. This contradiction proves that $\phi$ is one-to-one.

\section{Spectral characterization of Rank}

Theorem 2 and [10, Theorem 1] give spectral characterization of rank one operators. We now give a similar spectral characterization of operators of rank less than $k$ for an arbitrary positive integer $k$ (see also [14]).

Theorem 7.1. Let $X$ be a Banach space and $k$ a positive integer. For an operator $R \in \mathcal{L}(X)$, the following conditions are equivalent:

(i) $\operatorname{rank} R<k$.

(ii) $\bigcap_{1 \leq j \leq k} \sigma\left(T+\alpha_{j} R\right) \subseteq \sigma(T)$, for every $T \in \mathcal{L}(X)$ and every $k$ distinct scalars $\alpha_{j}(1 \leq j \leq k)$.

(iii) For every $T \in \mathcal{L}(X)$, there exists a compact subset $K_{T}$ of the complex plane, such that

$$
\bigcap_{1 \leq j \leq k} \sigma\left(T+\alpha_{j} R\right) \subseteq K_{T}
$$

for every $T \in \mathcal{L}(X)$ and every $k$ distinct complex numbers $\alpha_{j}(1 \leq j \leq k)$. Furthermore, these conditions are equivalent to condition (ii) or (iii) being satisfied for only operators $T$ of rank at most $k$. 
Proof. The implication (i) $\Rightarrow$ (ii) follows in exactly the same way as in Proposition 2.2 , and the implication (ii) $\Rightarrow$ (iii) is obvious.

We now assume that condition (iii) is satisfied for all operators $T$ of rank at most $k$. Following the proof of Proposition 2.2, by taking $T=0$, we conclude that $\sigma(R)$ is finite; indeed $\sigma(R) \subseteq\left\{0, c_{1}, \ldots, c_{k-1}\right\}$ for nonzero complex numbers $c_{j}(1 \leq j \leq k-1)$.

Next let $x \in X$ and $f \in X^{\prime}$ and define $G$ on $\left\{\zeta: \zeta^{-1} \notin \sigma(R)\right\}$ by $G(z)=$ $f\left((1-z R)^{-1} x\right)$. As in the proof of Proposition 2.2, we conclude that, for all $w$ large enough, the equation $G(z)=w$ has less than $k$ solutions. By Picard's Theorem, we get that $G$ has only removable singularities in the extended complex plane and so $G$ is a rational function $\frac{P}{Q}$ with $\operatorname{deg} P<k$ and $\operatorname{deg} Q<k$, and with the zeros of $Q$ included in the set $\left\{c_{j}^{-1}: 1 \leq j \leq k-1\right\}$. There are only a finite number of such polynomials $Q$, say $Q_{1}, Q_{2}, \ldots, Q_{N}$. Thus, we know that $\left(Q_{j} G\right)^{(k)}=0$ for $j=1,2, \ldots$, or $N$. We conclude that, for every $x \in X$ and $f \in X^{*}$, the function $G$ satisfies one of $N$ fixed (independent of $x$ and $f$ ) linear differential equations with polynomial coefficients and order $k$. As before, using the fact that a vector space is not a union of a finite number of proper subspaces, we see that $G$ satisfies one such differential equation for every $x$ and $f$. Using the fact that $G^{(m)}(0)=(m !) f\left(R^{m} x\right)$, we conclude that $R$ satisfies a polynomial equation $p(R)=0$ with $\operatorname{deg} p=k$.

If $\operatorname{rank} R \geq k$, then, by the standard decomposition of algebraic operators, there is a finite dimensional subspace $W$ of $X$, invariant under $R$ such that $\operatorname{rank}(R \mid W)=$ $k$. We choose a basis for $W$ so that

$$
R \mid W=R_{0} \oplus J_{1} \oplus \cdots \oplus J_{m}
$$

where $R_{0}$ is an upper triangular invertible $l \times l$ matrix with diagonal $\left\{c_{1}, \ldots\right.$, $\left.c_{l}\right\}$ and each $J_{j}$ is an upper triangular $t_{j} \times t_{j}$ nilpotent Jordan cell. Let $Z$ be a closed complement of $W$ in $X$ and let $T$ be an operator on $X$ such that $T \mid Z=0$ and

$$
T \mid W=T_{0} \oplus T_{1} \oplus \cdots \oplus T_{m},
$$

where $T_{0}=\operatorname{diag}\left(b_{1}, \ldots, b_{l}\right)$ with $b_{1}, \ldots, b_{l}$ distinct nonzero scalars, and where each other $T_{j}$ has all of its entries zero except the entry in the lower left corner where it is equal to $a_{j}$ with $\left|a_{1}\right|, \ldots,\left|a_{m}\right|$ distinct and nonzero.

We note that, for any scalar $\beta$, we have

$$
\sigma\left(T_{0}+\beta R_{0}\right)=\left\{b_{i}+\beta c_{i}: 1 \leq i \leq l\right\},
$$

and $\sigma\left(T_{j}+\beta J_{j}\right), 1 \leq j \leq m$, consists of the set of the $t_{j}$ th roots of $a_{j} \beta^{t_{j}-1}$. Thus

$$
\sigma(T+\beta R) \supseteq\left\{b_{i}+\beta c_{i}: 1 \leq i \leq l\right\} \cup\left(\bigcup_{1 \leq i \leq m}\left\{\zeta: \zeta^{t_{i}}=a_{i} \beta^{t_{i}-1}\right\}\right) .
$$

We observe that $k=\operatorname{rank}(R \mid W)=l+t_{1}+\cdots+t_{m}-m$, and that $\operatorname{rank} T \leq k$. We will show that, for all $\lambda$ large enough, there exists a set $B$ of $k$ distinct complex numbers such that $\lambda \in \bigcap_{\beta \in B} \sigma(T+\beta R)$. This would be a contradiction, implying that $\operatorname{rank} R<k$. Indeed we will show that this is satisfied for all complex numbers $\lambda$ outside a finite set to be determined later. For $\lambda \in \mathbb{C}$, let

$$
\beta_{i}=\frac{\lambda-b_{i}}{c_{i}}, \quad 1 \leq i \leq l,
$$


and let $\beta_{i 1}, \beta_{i 2}, \ldots, \beta_{i, t_{i}-1}$ be the distinct $\left(t_{i}-1\right)$ st roots of $\lambda^{t_{i}} a_{i}^{-1}$, for $1 \leq i \leq m$. Let

$$
B:=\left\{\beta_{i}: 1 \leq i \leq l\right\} \cup\left(\bigcup_{i=1}^{m}\left\{\beta_{i j}: 1 \leq j \leq t_{i}-1\right\}\right) .
$$

We show that

$$
\lambda \in \bigcap_{\beta \in B} \sigma(T+\beta R),
$$

and that, for all $\lambda$ outside a finite set, the elements listed in the definition of $B$ are all distinct and so $|B|=l+t_{1}+\cdots+t_{m}-m=k$. The first assertion (equation (3)) follows easily from the inclusion (1). Indeed if $\beta=\beta_{i}$ for some $1 \leq i \leq l$, then $\sigma(T+\beta R)$ contains $b_{i}+\beta_{i} c_{i}=\lambda$, and if $\beta=\beta_{i j}$ for some $1 \leq i \leq m$, then $\sigma(T+\beta R)$ contains all of the $t_{i}$ th roots of $a_{i} \beta_{i j}^{t_{i}-1}=\lambda^{t_{i}}$ and so contains $\lambda$. Next, we show that, for $\lambda$ outside some finite set $\Omega$, the set $B$ has $n$ distinct elements. Toward this end let

$$
\begin{aligned}
K & :=\left\{\frac{b_{i} c_{j}-b_{j} c_{i}}{c_{i}-c_{j}}: 1 \leq i, j \leq l, \quad c_{i} \neq c_{j}\right\}, \\
L_{s i} & :=\left\{z: a_{i}\left(z-b_{s}\right)^{t_{i}-1}-z^{t_{i}} c_{s}^{t_{i}-1}=0\right\}, \\
M_{i j} & :=\left\{z: a_{i}^{\left(t_{j}-1\right)} z^{t_{i}}-a_{j}^{t_{i}-1} z^{t_{j}}=0\right\}, \\
L & :=\bigcup\left\{L_{s i}: 1 \leq s \leq l, \quad 1 \leq i \leq m\right\}, \\
M & :=\bigcup\left\{M_{i j}: 1 \leq i, j \leq m, \quad i \neq j\right\},
\end{aligned}
$$

and

$$
\Lambda:=K \cup L \cup M
$$

The set $\Lambda$ is a finite set. We now show that, for $\lambda \notin \Lambda$, the set $B$ has $n$ distinct elements; i.e. all the elements listed in defining $B$ (equation (2)) are distinct. First if $\beta_{i}=\beta_{j}$ with $i \neq j$, then $\frac{\lambda-b_{i}}{c_{i}}=\frac{\lambda-b_{j}}{c_{j}}$ and so $\lambda \in K \subseteq \Lambda$. If $\beta_{s}=\beta_{i j}$ for some $1 \leq s \leq l, 1 \leq i \leq m, 1 \leq j \leq t_{i}-1$, then $\left(\frac{\lambda-b_{s}}{c_{s}}\right)^{t_{i}-1}=\lambda^{t_{i}} / a_{i}$, and so $\lambda \in L_{s i} \subseteq \Lambda$. If $\beta_{i p}=\beta_{j q}$ for some $i \neq j$, then upon raising each side to the $\left(t_{i}-1\right)\left(t_{j}-1\right)$ th power, we see that $\lambda \in M_{i j} \subseteq \Lambda$. This ends the proof.

\section{The Algebra of Regular operators on Banach lattices}

For a Banach lattice $X$, the algebra of regular operators, i.e., linear combinations of positive operators, is denoted by $\mathcal{L}^{r}(X)$. This is a Banach algebra if the norm of $T$ is defined to be the operator norm of $|T|$ (see [19]). Let $\mathcal{K}^{r}(X)$ be the closure of the set of finite rank operators in $\mathcal{L}^{r}(X)$ under the norm described above. The spectrum of an element $T$ in the algebra $\mathcal{L}^{r}(X)$ is called the order spectrum of $T$ and is denoted by $\sigma_{0}(T)$. Spectrum preserving linear maps between $\mathcal{L}^{r}(X)$ and $\mathcal{L}^{r}(Y)$ are characterized in [20]. This can be generalized to obtain a characterization of invertibility preserving linear maps. An examination of the proofs in this paper and in [20] establishes that Theorem 1.1, Corollary 1.2 and Theorem 3.4 remain valid for the algebras $\mathcal{L}^{r}(X)$ and $\mathbb{C} I+\mathcal{K}^{r}(X)$, and that Theorems 2.1 and 7.1 and Propositions 2.2 and 2.4 are valid where the spectrum is replaced by the order spectrum. In particular, we have the following 
Theorem 8.1. Let $X$ and $Y$ be complex Banach lattices. Every unital bijective invertibility preserving linear map from $\mathcal{L}^{r}(X)$ onto $\mathcal{L}^{r}(Y)$ or from $\mathbb{C} I+\mathcal{K}^{r}(X)$ onto $\mathbb{C} I+\mathcal{K}^{r}(Y)$ is a Jordan isomorphism. For every such a map $\phi$, either $\phi(T)=A^{-1}$ TA or $\phi(T)=B^{-1} T^{*} B$ where $A$ (respectively $B$ ) is a bounded invertible operator from $Y$ onto $X$ (respectively $X^{*}$ ).

\section{ACKNOWLEDGMent}

The author acknowledges inspiring conversations regarding the contents of this paper with Ciprian Foias. I would like to thank J. Zemanek for bringing reference [16] to my attention.

\section{REFERENCES}

1. B. Aupetit, Propriétés spectrales des algèbres des Banach, Lecture Notes in Math., vol. 735, Springer-Verlag, New York, 1979. MR 81i:46055

2. F. F. Bonsall and J. Duncan, Complete normed algebras, Springer-Verlag, Berlin, 1973. MR 54:11013

3. A. Brown and C. Pearcy, Structure of commutators of operators, Ann. of Math. (2) 82 (1965), 112-127. MR 31:2612

4. M.-D. Choi, D. Hadwin, E. Nordgren, H. Radjavi, and P. Rosenthal, On positive linear maps preserving invertibility, J. Funct. Anal. 59 (1984), 462-469. MR 86a:46071

5. H. DeGuang and B. V. Limaye, On a spectral characterization of rank one matrices, Linear Algebra Appl. 143 (1991), 1-6. MR 91i:15003

6. J. Dieudonné, Sur une généralisation du groupe orthogonal à quatre variables, Arch. Math. 1 (1949), 282-287. MR 10:5861

7. M. Eidelheit, On isomorphisms of rings of linear operators, Studia Math. 9 (1940), 97-105. MR 3:51e

8. A. Gleason, A characterization of maximal ideals, J. Analyse Math. 19 (1967), 171-172. MR 35:4732

9. I. N. Herstein, Topics in ring theory, Univ. of Chicago Press, Chicago, 1969. MR 42:6018

10. A. A. Jafarian and A. R. Sourour, Spectrum preserving linear maps, J. Funct. Anal. 66 1986, 255-261. MR 87m:47011

11. J. P. Kahane and W. Zelazko, A characterization of maximal ideals in commutative Banach algebras, Studia Math. 29 (1968), 339-343. MR 37:1998

12. I. Kaplansky, Algebraic and analytic aspects of operator algebras, Amer. Math. Soc., Providence, RI, 1970. MR 47:845

13. D. R. Larson and A. R. Sourour, Local derivations and local automorphisms of $\mathcal{B}(X)$, Proc. Sympos. Pure Math., vol. 51, part 2, Amer. Math. Soc., Providence, 1990, pp. 187-194. MR 91k:47106

14. V. Limaye, A spectral characterization of operators having rank $k$, Linear Algebra Appl. 143 (1991), 57-66. MR 92b:47004

15. M. Marcus and R. Purves, Linear transformations on algebras of matrices: The invariance of the elementary symmetric function, Canad. J. Math. 11 (1959), 383-396. MR 21:4167

16. M. Roitman and Y. Sternfeld, When is a linear functional multiplicative?, Trans. Amer. Math. Soc. 267 (1981), 111-124. MR 82j:46061

17. W. Rudin, Functional analysis, 2nd ed., McGraw-Hill, New York, 1991. MR 92k:46001

18. B. Russo, Linear mappings of operator algebras, Proc. Amer. Math. Soc. 17 (1966), 10191022. MR 33:6428

19. H. H. Schaefer, Banach lattices and positive operators, Springer-Verlag, Berlin, 1974. MR 54:11023

20. A. R. Sourour, Spectrum preserving linear maps on the algebra of regular operators, Aspects of Positivity in Functional Analysis, Elsevier, 1986, pp. 255-259. MR 88d:47049

21. _ A factorization theorem for matrices, Linear Multilinear Algebra 19 (1986), 141-147. MR 87j: 15028 
A. R. SOUROUR

22. E. C. Titchmarsh, The theory of functions, 2nd ed., Oxford Univ. Press, Oxford, 1939

23. W. Zelazko, acterization of multiplicative linear functionals in complex Banach algebras, Studia Math. 30 (1968), 83-85. MR 37:4620

Department of Mathematics and Statistics, University of Victoria, Victoria, B.C. V8W 3P4, CANADA 